\title{
Seamless Roaming in Wireless Networks for Video Streaming
}

\author{
Chih-Ming Chen ${ }^{1,2}$, Yung-Chang Chen ${ }^{1}$ \\ ${ }^{1}$ Department of Electrical Engineering \\ National Tsing Hua University \\ Hsinchu, Taiwan 300, R.O.C. \\ ycchen@ee.nthu.edu.tw \\ ${ }^{2}$ Chunghwa Telecom Co., Ltd.
}

\author{
Chia-Wen Lin \\ Department of Computer Science \& \\ Information Engineering \\ National Chung Cheng University \\ Chiayi, Taiwan 621, R.O.C. \\ cwlin@cs.ccu.edu.tw
}

\begin{abstract}
Roaming across multiple basic service sets (BSS) using access points (APs) in wireless LAN (WLAN) always pose considerable challenges, as it will cause heavy packet loss error. Especially for real-time video streaming, it will damage receiving video quality. In this paper, we combine the benefits of multiple descriptions coding (MDC) and multipath routing to maintain a best constant video quality despite packet loss when roaming in WLAN. By incorporating channel status detection mechanism to decide which channel will be selected or multiple channels will be used to take advantage of path diversity to deliver the streaming video content. The loss-rate and round-trip time are used to determine the channel status by using active probing. Simulation results indicate that the proposed method can significantly improve the quality of streamed video when mobile client roams in WLAN.
\end{abstract}

\section{INTRODUCTION}

The benefits for wireless applications are not only without the need of wire connections but also their mobile ability, especially for mobile computing devices such as smart phones and handheld devices. Because of its low cost, easy deployment, and flexible

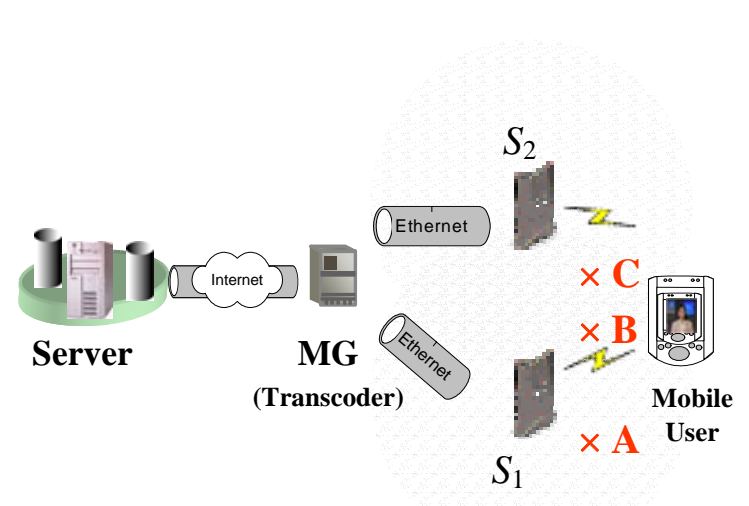

Fig. 1: Example of a three-tier streaming system. connectivity, WLAN is becoming widespread and leading to fastgrowing deployments in consumer homes.

A three-tier streaming system, as shown in Fig. 1, uses WLAN as an extension to the existing wired infrastructure, offering local end-point devices the convenience of wireless connections. The media gateway (MG) serves as a proxy/adaptation engine of MPEG-21 UMA architecture to adapt multimedia content for heterogeneous devices. Video transport over WLAN has its own characteristics different from that of wireline networks, such as high burst packet loss due to deep fading and handoff. To achieve a high level of acceptability, it needs to provide a reliable and efficient transmission. Multiple path transport schemes have been proposed in the past for wireless networks to increase connection reliability [1,2]. Multiple descriptions coding (MDC) has been shown to possess excellent error resilience capability for streaming video over error prone packet networks. In this paper, multiple description scalar quantization (MDSQ) [3] has been considered for use in the three-tier streaming system to enhance the robustness to deal with packet loss in WLAN, especially while mobile device with the need of handoff.

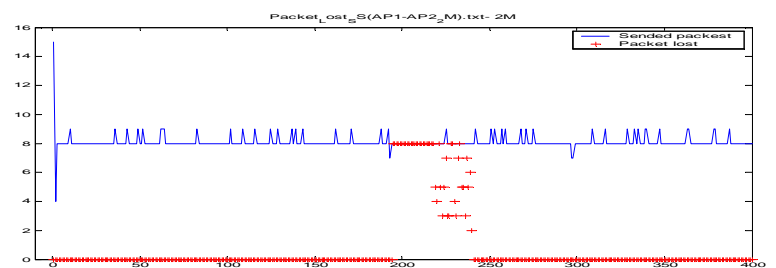

Fig. 2: Packet loss during handoff.

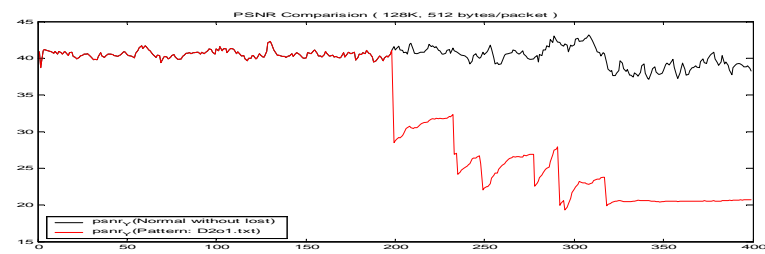

Fig. 3: PSNR distortion during handoff. 
In the WLAN infrastructure mode, a handoff to find another AP for a mobile device will occur when a channel cannot provide necessary connection quality guarantee. In general, a sequence of events must transpire for roaming. At first, the device must decide when to roam based on the factors such as signal strength and missed beacons. The second action is to decide where to roam, and then initiate a roam. The end, the client must resume the existing application session. After the long roaming duration, it performs a nomadic roaming and cause very heavy transmission data loss that will degrade the receiving video quality, as shown in Figs 2 and 3.

We use the ad-hoc mode in WLAN to provide a device to communicate with others simultaneously. As shown in Fig. 1, when a mobile device moves from station S1 to S2, The MG will know when the packets will be streamed only by S1 (S2) or by S1 and S2 with path diversity based on the conditions of both channels estimated through the status detection mechanism. For example, packets will be delivered only by S1 without using MDC when the mobile device staying around "A." And it will adopt multiple path diversity with MDC via S1 and S2 when the mobile user moves to "B." When it arrives at "C", packets will be delivered only by S2 without using MDC.

Low-latency wireless video over 802.11 networks using path diversity [4] was proposed to use multiple paths simultaneously or switch between multiple paths to provide significant benefits over conventional single AP (single path) systems. It generates five subsampled trace (AP1, AP2, Balanced, Site Selection, Oracle) to evaluate their performances. But it doesn't account for when and which trace must be used to get better overall performance based on channel conditions.

The rest of this paper is organized as the following. The basic idea of MDC transcoding will be reviewed in section II. The multipath streaming test bed is presented in section III. In section $\mathrm{IV}$, the channel status detection mechanism is given. In section $\mathrm{V}$, simulation results are provided. The conclusions are stated in section VI.

\section{MDC Transcoding}

MDC is a kind of joint source and channel coding methods. Its objective is to increase error resilience of data transmission under channel failures, by employing the diversity of channels. Data are encoded into several parts that are called "descriptions," and sent over multiple independent channels. The probability of failures of all channels is greatly decreased, so there will be a large probability to receive correct data from at least one channel, so that the decoder can estimate the original data with acceptable quality. MDC intentionally adds some redundancy among these descriptions, which, however, will consume more bandwidth. That is, MDC always has a trade-off between coding efficiency and error resilience.

MDSQ has been considered in this paper. As suggested in [3], the valid elements of index assignment matrices are designed to concentrate around the diagonal line of the matrix. Such an index assignment matrix is controlled by a redundancy parameter $\mathrm{k}$, and the width of diagonal lines of the matrix is set to $2 * k+1$. A larger $\mathrm{k}$ corresponds to smaller redundancy. That is, when $\mathrm{k}$ becomes larger, the central distortion D0 (when receiving both descriptions) will be smaller while the side distortion Ds (receiving only one description) will be larger, given the same bit rate. Fig. 4 shows an example of the index assignment of MDSQ used in our system.

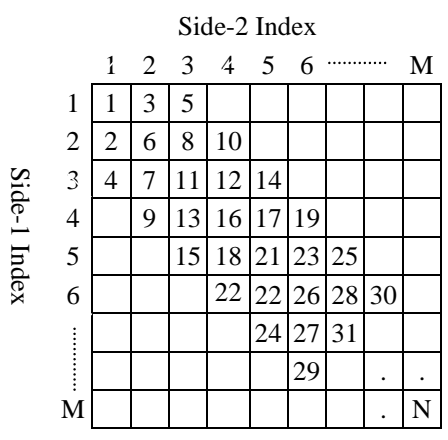

Fig. 4: Example of the index assignment for

\section{Multipath Streaming Test Bed}

As shown in Fig. 5, we build up a real-time multipath streaming test-bed that is composed of 4 personal computers to emulate a transcoder, two base stations (S1 and S2), and a client. In the test-bed, stations S1 and S2 are responsible for detecting if there is any client located in its communication zone, as well as reporting to the transcoder with channel characteristics information. According to the information, the transcoder will maintain a path list table that records which clients can be connected by the station. Paths are sorted according to their channel quality. The life time of the path will be updated after any information is received. After the life time, if the server doesn't receive any information for the client from any station, the status for the client will be destroyed, and the client will be marked as dis-connectable. Based on the path list table, the transcoder decides whether only one channel with excellent quality or two channels with good quality will be chosen to stream the video data to the client. The transcoder will send the data at a "Normal" or an "MD" mode. The channel status detection mechanism, as shown in Fig. 6, will be described later.

Pre-encoded bit streams that are encoded by a standard MPEG-4 encoder are stored in the server side. When the transcoder stays at the "MD" mode, a prestored bit stream will be read into the transcoder and transcoded into two descriptions using the MDSQ coding scheme for separately delivery via two paths simultaneously. Otherwise, at the "Normal" mode, the transcoder will forward the prestored bit stream directly to the client without any modification.

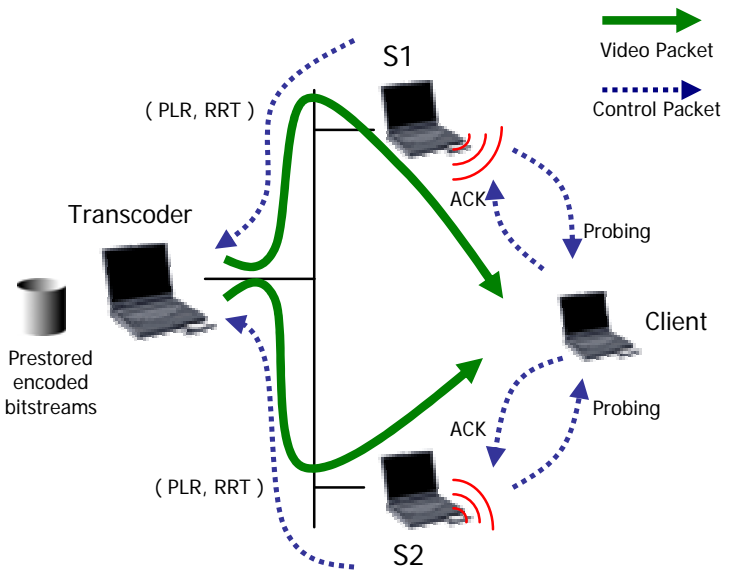

Fig. 5: Multipath Streaming test-bed. 


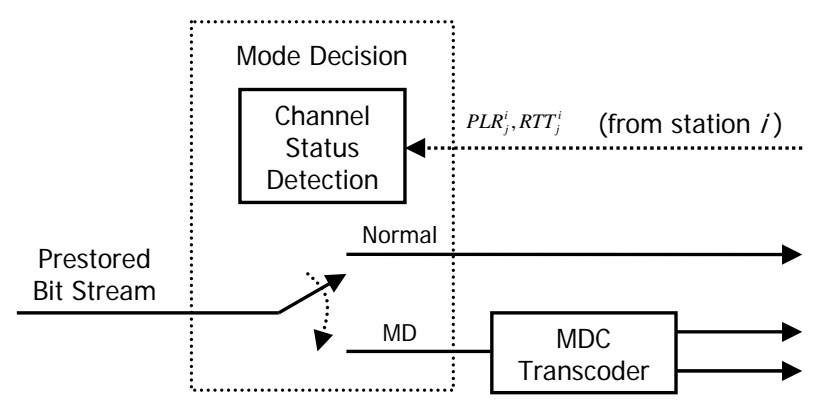

Fig. 6: Mode decision mechanism of the transcoder.

\section{Channel Status Detection}

In this section, the access mechanism of IEEE 802.11 is briefly reviewed. More detail can be found in [5]. We will then describe the proposed channel status detection mechanism which is used to estimate the channel conditions.

\section{A. Overview of IEEE 802.11 Access Mechanism}

In 802.11 WLAN networks, when a station wants to send data, it must sense the channel to determine whether another one is transmitting, If the medium is idle for an interval that exceeds the Distributed InterFrame Space (DIFS), the station will start its transmission. If the medium is busy, it will continuously monitor the channel until it is idle for a DIFS, and then the station initiates a random backoff interval that is used to initiate the backoff timer for the station. The timer counts down as the medium is sensed idle, but stopped counting when any transmission is detected, and will continue counting down when the medium is sensed idle again for more than a DIFS. When the backoff timer reaches zero, the station will start its transmission. When the receiver receives the data frame successfully and waits for a Short InterFrame Space (SIFS), it will feed back an acknowledgement (ACK) to the sender. If the sender does not receive the ACK within a specified timeout interval or detects another transmission in the channel, the sender will retransmit the frame again according to the backoff rule. For any transmission, the backoff interval is uniformly chosen in $[0$, $\mathrm{CW}-1$ ] where $\mathrm{CW}$ is the contention window that will be doubled at each retransmission. The standard allows a maximum of seven retransmissions before the data is dropped.

\section{B. Channel Status Detection}

According to the access mechanism of IEEE 802.11, if the condition of a channel is poor, the transmit station needs more retransmissions to successfully send the video data to the receiver, leading to an increase of delay time for the data to arrive at the receiver side. Furthermore it will have a higher loss rate. This two attributes also have been taken as congestion indicators in many existing congestion control schemes [6,7]. So we use the delay time and loss rate to estimate the channel condition, no matter they are made by network congestion or the unreliable transmission media.

The stations use UDP to send the probe frames every 1 second and receive the ACK frames from the client. Only a departure timestamp and sequence number are carried in the probe frames and also in the ACK frames. For the probing, it is difficult to measure the delay time accurately unless the clock times of station and client are well synchronized. Using the round-trip time (RTT) to replace the one-way delay to estimate the channel condition is more reliable, because the receiver sends back the ACK frame immediately over the same channel. In this paper, the stations use a sliding window to calculate $\overline{R T T_{k}^{i}}$ and $\overline{P L R_{k}^{i}}$ of each channel between the stations and the client, and report to the transcoder as references to decide which channels will be used, where $\overline{R T T_{k}^{i}}$ and $\overline{P L R_{k}^{i}}$ denote the averaged RTT and the packet loss rate in an interval C consisting of a constant number of packets, as shown in (1) and (2), respectively.

$$
\begin{aligned}
& \overline{R T T_{k}^{i}}=\frac{1}{C} \sum_{j=k-C+1}^{k} R T T_{j}^{i} \\
& \overline{P L R_{k}^{i}}=\frac{1}{C} \sum_{j=k-C+1}^{k} P L_{j}^{i} \\
& \max R T T^{i}=\operatorname{Max}\left(R T T_{k}^{i}, \max R T T^{i}\right)
\end{aligned}
$$

where $R T T_{j}^{i}$ stands for RTT of the $j$-th packet through the $i$-th channel, $P L_{j}=1$ indicates the $j$-th packet through the $i$-th channel gets lost, and $R T T_{j}^{i}=\max R T T^{i}$ if $P L_{j}^{i}=1$.

The proposed algorithm of the channel status detection used in the transcoder is listed as follows:

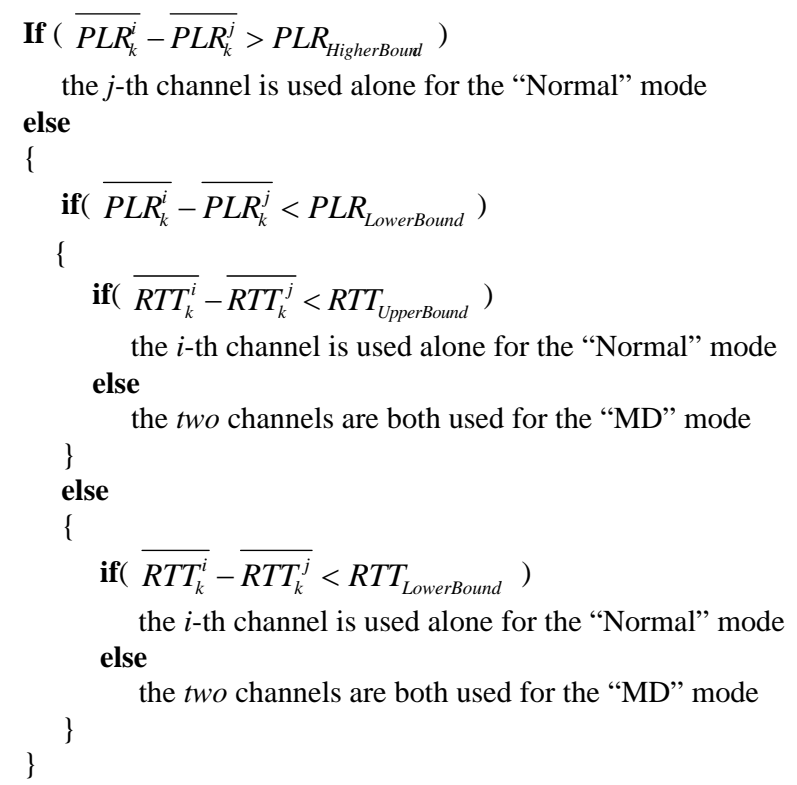

where we just consider the case of only two channels in order to reduce the complexity of description and implementation, but it can be generalized to the cases of more channels. In the algorithm, we don't need to identify the exact quality of each channel accurately, but only need to know the relative qualities of the two channels.

\section{V.EXPERIMENTAL RESULTS}

In order to construct a roaming environment in a lab environment, the stations are physically separated by about 30 meters and their transmitting power is reduced to $25 \%$. A factory default transmission retry limit of 7 is configured for the stations. 

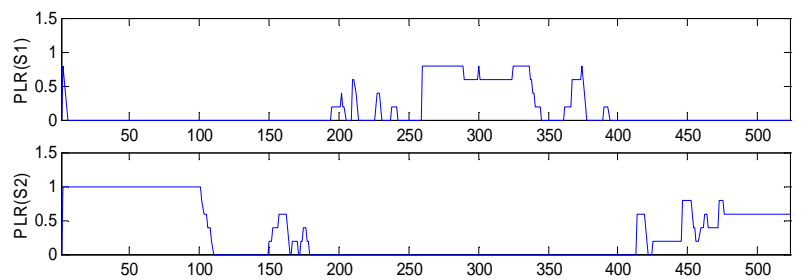

(a)
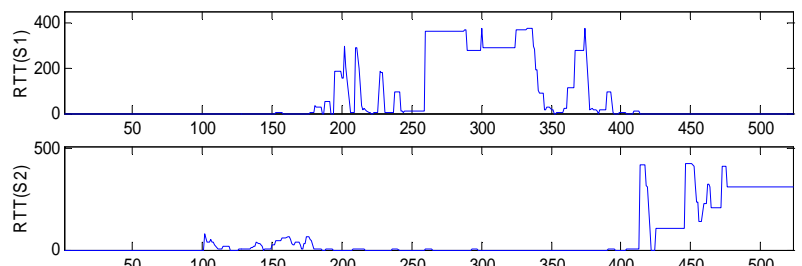

(b)

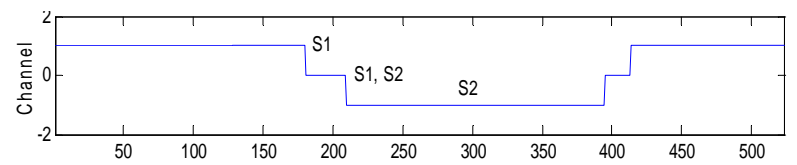

(c)

Fig. 7: (a) $\overline{P L R}$, (b) $\overline{R T T}$ of the channels by $S_{1}$ and $S_{2}$, and (c) channel selection result.

The QCIF 4:2:0 test sequences are encoded by an MPEG-4 coder at $256 \mathrm{Kbps}$ and $30 \mathrm{fps}$ for experiments. The first frame is coded as an I-frame, and all subsequent frames are coded as P-frame. The video packet length is 512 bytes. We simulate a roaming between stations S1 and S2 with a scenario that a client moves from station S1 toward station S2 and then moves back to S1 at the walking speed. Fig. 7 shows the $\overline{P L R}$ and $\overline{R T T}$ traces of the channels by S1 and S2, and the channel selection result by the transcoder. At time instance 180 in Fig. 7, the channel status detection mechanism detects the $\overline{P L R}$ and $\overline{R T T}$ of the first path by S1 are going worse and finds that the second path by S2 has good condition, so it decides to use the "MD" mode to deliver data to the client through both paths. Then at time instance 210, the first path is even worse than the second path, so the path for delivery is fully switched to the second path by S2. The situation is similar during time 395 to 414 when the client happens to roam from S2 to S1. We adopt the zero-motion replacement error concealment for lost video data. Fig. 8 compares the performance of our proposed method for two cases. In the first case, the transcoder is configured in the "MD" mode which transcodes an incoming bit stream into two descriptions with the same data rate (256Kpbs). In the second case, the transcoder transcodes the bit stream without quality loss but the data rate in increased by $20 \%$. In this case, it will not lead to any "drifting" when switching from the "Normal" mode to the "MD" mode or vice versa since there is no video quality loss after transcoding. Fig. 8 also shows that the proposed MD coding with multipath transmission can greatly reduce the distortion caused by roaming with transition, even there still are some unavoidable packet losses at the "MD" mode. The average PSNR performance improvement for roaming over WLANs with the proposed method at the same data rate is about $5.4 \mathrm{~dB}$ for the channel condition shown in Fig. 7, whereas the coding performance degradation due to the MD coding for clean channels is about $0.75 \mathrm{~dB}$.

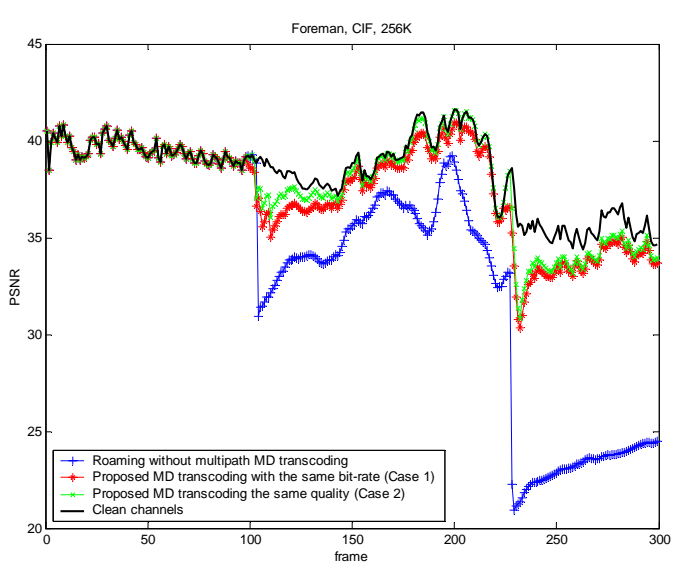

Fig. 8: PSNR performance comparison.

\section{CONCLUSION}

We proposed to combine MDC and multipath routing to tackle packet loss while a mobile client roams in WLAN. With path diversity, a two-step "soft" roaming method has been proposed. When a mobile client roams from one station to another, the proposed transcoder will try to find whether there is another path with an acceptable channel condition to be used simultaneously for path diversity and takes as "MD" mode. Then the mobile client will change its connection to the other one completely and switch to the "Normal" mode again. In order to find when to switch between the two modes, we proposed to use the feedback information: round-trip time and the packet loss rate to estimate the channel condition, no matter they are made by network congestion or the unreliable transmission media. Finally, we have established a real-time multipath streaming test-bed to verify the performance of the proposed scheme. Experimental results show that the use of path diversity as temporary step for roaming can provide significant benefits compared to the roaming among access points (APs). The proposed method also can be extended to the cellular applications, such as the $3 \mathrm{G}$ systems, to achieve reliable roaming of mobile users in a wider WLAN area.

\section{REFERENCES}

[1] Y. Wang, S. Panwar, S. Lin, and S. Mao, "Wireless video transport Using diversity: Multiple description vs. layered coding,” in Proc. IEEE ICIP, pp. 21-24, Oct. 2002.

[2] V. K Goyal, "Multiple description coding: Compression meets the network," IEEE Signal Processing Magazine, vol. 18, no.5, pp. 7493, Sept. 2001

[3] V. A. Vaishampayan, "Design of multiple description scalar quantizers,” IEEE Trans. Information Theory, vol. 39, no. 3, pp. 821834, May 1993.

[4] A. Miu, J. G. Apostolopoulo, W.-T. Tan, and M.l Trott, "Lowlatency wireless video over 802.11 networks using path diversity," in Proc. IEEE ICME, pp. 441-444, Aug. 2003.

[5] IEEE P802.11. Standard for Wireless LAN Medium Access Control (MAC) and Physical Layer (PHY). Nov. 1997

[6] K. Tan, Q. Zhang, and W. Zhu, "An end-to-end rate control protocol for multimedia streaming in wired-cum-wireless environments," in Proc. IEEE ISCAS, pp. 836-839, May 2003.

[7] S. Cen, P. C. Cosman, and G. M. Voelker, "End-to-end differentiation of congestion and wireless losses", IEEE/ACM Trans. Networking, vol. 11, pp. 703-717, Oct. 2003 